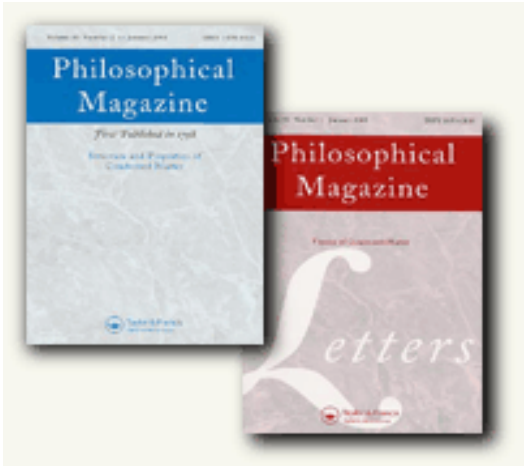

\title{
Atomistic study of structure and stability of thin Ni films on Fe surfaces
}

\begin{tabular}{|r|l|}
\hline Journal: & Philosophical Magazine \& Philosophical Magazine Letters \\
\hline Manuscript ID: & TPHM-09-Jun-0275.R1 \\
\hline Journal Selection: & Philosophical Magazine \\
\hline Date Submitted by the & 26-Aug-2009 \\
\hline Complete List of Authors: & $\begin{array}{l}\text { Hashibon, Adham; Fraunhofer Institute for Mechanics of Materials; } \\
\text { Uni Karlsruhe, izbs } \\
\text { Schravendijk, Pim; Technische Universitaet Darmstadt, Center of } \\
\text { Smart Interfaces, Computational Methods } \\
\text { Elsaesser, Christian; Fraunhofer Institute for Mechanics of Materials } \\
\text { IWM; Uni Karlsruhe, izbs } \\
\text { Gumbsch, Peter; Fraunhofer Institute for Mechanics of Materials } \\
\text { IWM; Uni Karlsruhe, izbs }\end{array}$ \\
\hline Keywords: & $\begin{array}{l}\text { bonding, density-functional theory, molecular dynamic simulations, } \\
\text { Structural transitions, thin films }\end{array}$ \\
\hline Keywords (user supplied): & \begin{tabular}{l} 
\\
\hline
\end{tabular} \\
\hline
\end{tabular}

\section{S ScholaroNE \\ Manuscript Central}


Philosophical Magazine

Vol. 00, No. 00, 00 Month 200x, 1-13

\title{
RESEARCH ARTICLE
}

\section{Atomistic study of structure and stability of thin Ni films on Fe surfaces}

\author{
Adham Hashibon $^{a, b *}$, Pim Schravendijk ${ }^{b \dagger}$, Christian Elsässer $^{a, b}$, and Peter Gumbsch ${ }^{a, b}$ \\ ${ }^{a}$ IZBS, University of Karlsruhe, \\ Kaiserstr. 12, 76131 Karlsruhe, Germany \\ ${ }^{b}$ Fraunhofer-Institute for Mechanics of Materials IWM, \\ Wöhlerstr. 11, 79108 Freiburg, Germany \\ (Received 00 Month 200x; final version received 00 Month 200x)
}

\begin{abstract}
The adhesion and residual strain of $\mathrm{Ni}$ thin-film coatings on $\gamma$-Fe and $\alpha$-Fe substrates are investigated by $a b$ initio calculations using density functional theory (DFT) in the local density approximation (LDA), and by an empirical Finnis-Sinclair type interatomic potential utilizing angle dependent terms [Mishin et al., Acta Mat. 53 (2005), 2029]. The results from the DFT and empirical potentials agree for strained coherent interfaces. The phase stability and structural transitions are studied for incoherent interfaces via molecular dynamics and static relaxation methods. It is found that the transition in thin Ni films from bcc to fcc structure occurs for 3-4 monolayers and is accompanied by a reorientation of the Ni film with respect to the Fe substrate.
\end{abstract}

\section{Introduction}

Thin film systems play an important technological role due to their unique mechanical, chemical, and physical properties; thin film systems are found in magnetic storage devices, used as wear resistant coatings, as corrosion protection layers or as thermal barriers. Film systems with specifically tuned properties are designed and used for various applications. In coating applications for example, the main goal is to obtain strong and highly wear and corrosion resistant films that adhere strongly to the substrates. For magnetic storage applications, the main requirement is to design films with stable magnetic structures that do not change due to thermal fluctuations during the device life time. The adhesion, the resilience against wear, and the magnetic properties are closely dependent on the atomistic structure and chemical bonding at the interface between the substrate and the film as well as on the atomistic structure of the film itself.

The adhesion at the interface depends on the interatomic interactions between the substrate and film. In a coherent interface system, where the substrate and film have the same in-plane lattice parameters, the adhesion depends primarily on the chemical bonding [1-3]. Yet, perfect coherency is seldomly encountered, as in general the lattice parameters do not perfectly match, and additional structural terms resulting from the lattice misfit at the interface has to be taken into account. If the interaction between the substrate and film materials is strong enough, then there will be a certain degree of structural matching, or compliance, of the film with

\footnotetext{
*Corresponding author. Email: adham.hashibon@iwm.fraunhofer.de

${ }^{\dagger}$ Current address: Technische Universität Darmstadt, Center of Smart Interfaces, Computational Methods, Petersenstraße 32, 64287 Darmstadt

ISSN: 1478-6435 print/ISSN 1478-6443 online

(C) 200x Taylor \& Francis

DOI: $10.1080 / 1478643 Y Y x x x x x x x x$

http://www.informaworld.com
} 
the substrate. This leads on the one hand to a stronger adhesion and lower excess energy of the interface, but on the other hand it is accompanied by residual stresses and their corresponding strains in the thin film. The excess energy due to these stresses may or may not exceed the gain in energy due to bonding. The balance between the bonding and stresses determines the amount of compliance of the film to the substrate and its phase stability. Perfect compliance of the film results in a pseudomorphic phase, where the film assumes the same lattice structure as the substrate [4].

Residual stresses in thin films also originate from manufacturing processes, e.g., electroplating, and they may also result at normal operating conditions, for instance when high temperature gradients exist, causing different thermal expansions of substrate and film and thus a bending of the film. The coupled effect of adhesion and strain on the macroscopic properties of the thin film system is of crucial importance to the successful design and operation of the device. In general, stresses in thin films are undesired as they may cause fatigue, delamination, and a decrease in performance in corrosive environments. On the other hand, for magnetic applications, the interplay between bonding and strain leads to various magnetic properties and phases, and it is therefore an important factor in the design of these systems (see, e.g., Refs $[3,5]$ ).

Fe-based magnetic thin film systems are most widely used for their magnetic properties, either in single-layer or in multilayer structures (for a recent review, cf. $[6])$. In most cases the Fe-containing layers need protection against corrosion, and $\mathrm{Ni}$ is often used for protective coating [6]. Compared to other thin film systems, the case of Ni films deposited on Fe substrates has been less studied so far, in spite of its relevance for mechanical and magnetic applications. In particular, theoretical studies for the interface structure and adhesion for $\mathrm{Ni}$ on $\mathrm{Fe}$ are rare [6]. In the current study we investigate the thermal and mechanical stability of thin Ni films on Fe substrates at temperatures ranging from $0 \mathrm{~K}$ up to $1200 \mathrm{~K}$.

The bcc phase of $\mathrm{Ni}$, which does not exist in nature, has been shown by firstprinciples DFT calculations $[7,8]$ to correspond to a metastable phase with an equilibrium lattice constant of $a^{b c c N i}=2.78 \AA$, which has a small lattice mismatch with bcc $\alpha$-Fe $\left(a^{\alpha-F e}=2.866 \AA\right.$ A, cf., e.g., Ref. [8]). Therefore it is expected that bcc Ni may grow as a metastable phase on $\alpha$-Fe (001). This bcc Ni phase was obtained by means of molecular beam epitaxy by Heinrich et al. [9, 10] and by Wang et al. [11]. These experimental results suggest that bcc Ni can be grown pseudomorphically on the bcc $\mathrm{Fe}(001)$ surface with up to 3 [10] or 6 [11] Ni (100) monolayers, depending on the substrate conditions. Above this thickness the lattice constant expands by $2 \%$ and develops a $c(2 \times 2)$ surface reconstruction. The unreconstructed and strained bcc Ni films are found to be non-magnetic, while the reconstructed films are magnetic with four-fold in-plane magnetic anisotropy and a magnetic moment of approximately $0.4 \mu_{B}$ /atom for a $6 \mathrm{~nm}$ bcc Ni film on $\mathrm{Fe}$ (001). This demonstrates the close relationship between the atomistic structure and the magnetic functional properties of the thin film. Moreover it offers a means for tuning these properties by controlling the growth mode.

An investigation of the structural changes in MBE grown Ni/Fe(001) multilayers, using RHEED and X-ray diffraction by Kamada et al. [12, 13], indicated that the structure of up to $3 \mathrm{ML}$ of $\mathrm{Ni}$ is body-centered-tetragonal (bct) instead of the expected bcc structure (i.e., bct with $\mathrm{c} / \mathrm{a}=1$ ), and that it has an enhanced moment of $0.8 \mu_{B}$. Above $3 \mathrm{ML}$, the structure becomes more disordered, and seems to resemble that of the reconstructed $c(2 \times 2)$ structure. Mijiritskii et al. [14, 15] have suggested, based on a detailed study of the angle dependence of the RHEED patterns, that this apparent surface reconstruction corresponds instead to the fcc 
$\mathrm{Ni}$ phase (i.e., bct with $\mathrm{c} / \mathrm{a}=\sqrt{2}$ ) consisting of a mosaic of four different $\mathrm{Ni}(110)$ domains. The same possibility was also considered by Heinrich et al. [9, 10]. At the thickness where these extra diffraction patterns appear, a martensite-like bcc-Ni to fcc-Ni transformation may have taken place. Gutierrez et al. [16] observed for an $\mathrm{Fe} / \mathrm{Ni}$ multilayer a bcc Ni structure, albeit with weak reconstruction features. This slight discrepancy in the exact description of the structural phases can be effectively investigated using atomistic simulations via the Finnis-Sinclair (FS) or the related embedded-atom-method (EAM) interatomic potentials. We therefore consider here two types of interfaces between very thin films of up to 25 layers of either bcc or fcc $\mathrm{Ni}$ on $\mathrm{Fe}$. In the first type films of $\mathrm{Ni}$ are assumed completely compliant, i.e., pseudomorphic with $\mathrm{Fe}$, and in the second type, more general incoherent interfaces are considered as initial conditions for the structural optimization.

Experimental data $[11-15,17]$ suggests that thin films of Ni composed of up to 3 or 4 layers are probably stable in the bcc structure. Systems with more than 4 layers exhibit a structural transformation of the bcc Ni into an intermediate, deformed fcc phase. The nature of this intermediate phase, and the specific number of layers at which the transformation occurs, are not entirely understood. Atomistic molecular dynamics and static relaxation simulations, for instance with the interatomic potential of Mishin et al. [8], enable an atomistic insight into the structural changes. We therefore perform such simulations of the structure and stability of such Ni films on Fe substrates and investigate the structural transformations.

This work is divided into two parts. In the first part, the completely compliant pseudomorphic thin Ni films on both ferrite, $\alpha$-Fe, and austenite, $\gamma$-Fe, are examined for their residual stresses and adhesion using first-principles density-functional theory (DFT) calculations in the local density approximation (LDA). For comparison, we also consider pseudomorphic interfaces between $\mathrm{Cr}$, which is as well as $\mathrm{Ni}$ used in coating applications, and Fe. In the second part, the methods of molecular dynamics and static relaxation based on empirical interatomic potentials are used to study both pseudomorphic and incoherent $\mathrm{Ni}$ overlayers on Fe. The Fe-Ni potential developed by Mishin et al. [8], which is an extension of the embedded atom method (EAM) by additional angle-dependent terms (the EAM-ADP potential) is used for this study. This potential does not allow modeling of magnetism, although it does include magnetic contributions to the total energy [8]. Magnetic effects are thus not explicitly considered.

\section{Computational methods}

\subsection{First-principles DFT calculations}

The computational first-principles method used in the present study is based on density functional theory $[18,19]$ and the local-density approximation for exchange and correlation (LDA) [20, 21], employing norm-conserving pseudopotentials [22] and a mixed basis of localized orbitals and plane waves [23-29]. The pseudopotentials were constructed from all-electron valence states for free atoms according to Vanderbilt [30]. Plane waves up to the cutoff energy $E_{p w}=16$ Rydberg were used $(1$ Rydberg $=13.606 \mathrm{eV})$. Localized functions confined to atom-centered spheres with radii $R_{l o}=2.5 \mathrm{Bohr}(1 \mathrm{Bohr}=0.529 \AA)$ were employed for d valence states. A Monkhorst-Pack $8 \times 8 \times 2$ k-point mesh and a Gaussian broadening by $0.2 \mathrm{eV}$ were used $[24,31]$. These parameters led to convergence of differences in surface energies to less than $0.05 \mathrm{~J} / \mathrm{m}^{2}$.

For the current study, spin polarization is not taken into account. Although this prevents the extraction of the magnetic properties of these interfaces from the 
results, it does at least allow for a direct comparison between the DFT calculations and the non-magnetic atomistic simulations using the ADP-EAM potential.

\section{2. $\quad$ Supercell models}

For the DFT calculations, small coherent interface systems are used. The interface systems of the thin $\mathrm{Ni}$ and $\mathrm{Cr}$ films on both $\alpha$-Fe and $\gamma$-Fe substrates are modeled by slab supercells, consisting of seven atomic (110) and (111) layers for $\alpha$-Fe and $\gamma$-Fe, respectively. The thin fcc Ni films are composed of eight atomic (111) layers, while the bcc Cr films are composed of seven atomic (110) layers. Each atomic layer is represented by one atom per surface unit cell. Periodic boundary conditions parallel to the surface and normal to the interface are applied, such that two equivalent interfaces are present in the systems. The in-plane lattice constants of $\mathrm{Ni}$ and $\mathrm{Cr}$ films are fixed due to the interaction with the substrate, while the out-of-plane lattice parameter is free to adjust to the value that minimizes the free energy of the system, usually tending to keep the volume of the unit cell approximately constant (Poisson effect). This is a general phenomenon which is encountered in solid-solid and even in solid-liquid interfaces [32, 33].

\subsection{Molecular dynamics and static relaxations}

For the atomistic simulations, an EAM-type potential with additional angledependent terms for Fe-Ni developed by Mishin et al. [8] was used. The angledependent terms are added to simulate the bcc phase more accurately. Due to the absence of magnetism in the potential, but the inherent presence of magnetic effects in the experimental and ab-initio data to which the potential was parametrized, this potential tends to show correct bonding in those configurations where a magnetic bonding interaction is present, but it shows over-bonding in those configurations where there is no or a negative contribution of the magnetic interaction to the bonding. The presence of magnetic effects in thin $\mathrm{Ni}$ films on $\mathrm{Fe}$ is therefore expected to be reasonably well mimicked.

\subsection{Ideal work of separation, excess strain and interface energies}

The ideal work of separation $W_{\text {sep }}$ is defined as the energy difference per unit area between an interface system and one where the two surfaces are completely separated:

$$
W_{\text {sep }}=\frac{E_{\text {film }}+E_{\text {substrate }}-E_{\text {interface }}}{2 A}
$$

where $A$ is the interface area, $E_{\text {film }}$ and $E_{\text {substrate }}$ are the energies of separated film and substrate, and $E_{\text {interface }}$ is the total energy of the interface system. No relaxation of atomic positions is taken into account. The validity of this approach has been extensively discussed elsewhere $[34,35]$. $W_{\text {sep }}$ specifies how much energy needs to be invested in the system to cause a separation of the interface [36]. The work of separation calculated in this manner gives direct information on the chemical bonding at the interface.

The excess strain energy $\gamma_{\epsilon}$ is defined as the energy difference of a strained bulk crystal with exactly the same strain as in the interface system, and an unstrained bulk crystal. If the strain components in the film are $\epsilon_{x}$ and $\epsilon_{y}$, parallel to the interface in a coherent configuration, the resulting strain energy per monolayer 


\section{Page 5 of 23 \\ Philosophical Magazine \& Philosophical Magazine Letters}

and unit area is:

$$
\gamma_{\epsilon}=\frac{E^{b u l k}\left(\epsilon_{x}, \epsilon_{y}\right)-E^{b u l k}(0)}{n A_{\epsilon}}
$$

where $E^{\text {bulk }}\left(\epsilon_{x}, \epsilon_{y}\right)$ is the total energy of a strained film, taking into account the relaxation in the direction normal to the interface. $E^{b u l k}(0)$ is the energy of an unstrained bulk crystal (which we consider as the reference chemical potential for the film), $n$ is the number of layers, and $A_{\epsilon}$ is the area of a strained system [37]. Hence, the work of separation represents the chemical contribution to the interfacial bonding, while the structural contributions are given by the excess strain energy $[1$, $2]$. The adhesion of the interface is then analyzed by considering both of these two quantities.

The excess interface energy of the system is expressed as:

$$
\gamma=\left(E-\Sigma_{i} n_{i} \mu_{i}\right) / 2 A
$$

where $E$ is the total energy of the system, $n_{i}$ and $\mu_{i}$ are the number of atoms and chemical potential of species $i$ (the latter referred to the bulk phases of $\mathrm{Cr}, \mathrm{Fe}$, and $\mathrm{Ni})$.

\section{Results and Discussion}

\subsection{Interface adhesion and mechanical stability of coherent interfaces}

In this section, ideal coherent interface models, in which the film is assumed to fit exactly onto either bcc or fcc substrates of bcc $\alpha$-Fe and fcc $\gamma$-Fe, respectively, are considered. It is expected therefore that unless the lattice parameters of the substrate and film match exactly, a finite amount of strain will be present in the system, which in turn will affect the mechanical interface stability. In this study, the mechanical stability of the system is investigated via the ideal work of separation, $W_{\text {sep }}$, and the excess strain energy per monolayer in the film, $\gamma_{\epsilon}$, as described in section 2.4.

Four different interface models between $\mathrm{Cr}, \mathrm{Ni}$ and $\mathrm{Fe}$ are considered, thin films of bcc $\mathrm{Cr}(110)$ and fcc $\mathrm{Ni}(111)$ are deposited onto either the ferrite, bcc $\alpha$-Fe(110), or the austenite, fcc $\gamma$-Fe(111), substrates. Hence bcc/bcc, fcc/bcc, and fcc/fcc hetero-phase interface models are considered. In the case of bcc/bcc and fcc/fcc systems, the coherent interface was created by straining the lattice parameter of the top layer, taken to be the $\mathrm{Cr}$ or $\mathrm{Ni}$ film, to the bcc and fcc lattice parameters of Fe, respectively. In the case of fcc (111) $\mathrm{Ni}$ on $\alpha$-Fe, the fcc unit cell of the film is strained to fit the underlying bcc unit cell of the substrate, but the same ABC stacking of (111) planes as in the fcc system is assumed. Similarly for the case of bcc (110) Cr on $\gamma$-Fe, the bcc (110) unit cell of $\mathrm{Cr}$ is fitted to the fcc (111) unit cell of $\gamma-\mathrm{Fe}$, but the AB stacking of (110) planes is assumed. The NishiyamaWassermann orientation relationship is used for the bcc-fcc systems, where the $\langle\overline{11} 2\rangle$ direction on the fcc (111) surface, and the $\langle 1 \overline{1} 0\rangle$ direction on the bcc (110) surface are parallel, and similarly the $\langle 1 \overline{1} 0\rangle$ direction on the fcc (111) surface and the $\langle 001\rangle$ direction on the bcc surface are aligned. The lattice parameters of the $\mathrm{Ni}$ or Cr layer along these directions were adjusted to the corresponding ones of the Fe substrate. The resulting misfit strains are given in table 2. The in-plane strain, $\epsilon_{x}$ and $\epsilon_{y}$, are smallest for the systems with the same lattice structures, namely for fcc $\mathrm{Ni}$ on fcc $\mathrm{Fe}$, and for bcc $\mathrm{Cr}$ on bcc Fe. In these systems relaxation effects are 
therefore expected to be small. On the other hand for the pseudomorphic systems of fcc $\mathrm{Ni}$ on bcc Fe, and bcc $\mathrm{Cr}$ on fcc Fe, the in-plane strains are large, implying that large deformations should be expected in the relaxed systems.

The interface separation energy, i.e., the negative of $W_{s e p}$, as a function of the separation $\Delta$ between the film and substrate is shown in Figure 1, where the open symbols are data points from the non-magnetic DFT-LDA calculations and the lines are fits of the universal equation of state (cf., e.g., [38]). The interface separation energy calculated using the Fe-Ni EAM-ADP potential of Mishin et al. [8] is marked by filled symbols. The agreement, as can be seen, is very good, in particular since such $\mathrm{Fe} / \mathrm{Ni}$ separation curves were not part of the fitting database [8]. This demonstrates the consistency of the non-magnetic DFT-LDA and the empirical EAM-ADP for describing the interface bonding. The interface energy at equilibrium separation $\Delta$, i.e., $W_{\text {sep }}$, and the excess energy per layer of the film due to coherency strains, $\gamma_{\epsilon}$, are given in Table 3. From Figure 1 and Table 3 it is obvious that $\mathrm{Cr}$ generally bonds better than $\mathrm{Ni}$ to both phases of $\mathrm{Fe}$, regardless of the strain energy, with $W_{\text {sep }}$ of Cr/Fe being about $1 \mathrm{~J} / \mathrm{m}^{2}$ larger than that of $\mathrm{Ni} / \mathrm{Fe}$. Nevertheless, pure Ni is also found to bond rather well to Fe. The chemical contribution to the interface energy is rather independent of the geometry of the interface, since nearly the same $W_{\text {sep }}$ is obtained for fcc Ni on either fcc or bcc $\mathrm{Fe}$, and for bcc $\mathrm{Cr}$ on either fcc or bcc Fe. This supports our assumption that the ideal work of separation $W_{\text {sep }}$ primarily gives information on the bonding at the interface $[34,35]$. However, as given in Table 3 the excess strain energy due to the coherency is large for the cases of fcc $\mathrm{Ni}$ on bcc Fe, and for bcc Cr on fcc Fe. This indicates that in these systems the $\mathrm{Ni}$ and $\mathrm{Cr}$ are not stable in their respective bulk equilibrium structures, which was already expectable from the misfit strains in Table 2. On the other hand, the strain energy in the bcc/bcc and fcc/fcc systems is much smaller, implying that indeed in these system the thin films are stable. In particular for the systems with the same structure, $\mathrm{Ni}$ on $\gamma$-Fe and $\mathrm{Cr}$ on $\alpha$-Fe, the residual strains are small. Table 2 shows the in-plane strains $\left(\epsilon_{x}\right.$ and $\left.\epsilon_{y}\right)$ calculated from the lattice parameters in Table 1, and the corresponding Poisson relaxation. Clearly the systems with the smallest strain energy per monolayer are the ones with the smallest coherency strains. The larger strain energy for $\mathrm{Cr}$ on $\gamma$-Fe indicates either that bcc $\mathrm{Cr}$ will be unstable on $\mathrm{Fe}$ and will probably dewet, leaving perhaps a few pseudomorphic monolayers, similar to what has been observed for $\mathrm{Cu}-\mathrm{Ta}$ recently by both DFT calculations and molecular dynamics simulations [35, 39], or that it would assume a pseudomorphic bcc structure.

\subsection{Structure and stability of Ni overlayers on $\alpha-F e(100)$}

The work of separation calculations in the preceding section demonstrated a very good agreement between the EAM-ADP interatomic potential for Fe-Ni of Mishin et al. [8] and the non-magnetic DFT-LDA calculations. In this section, the interatomic potential is used to move away from the constraints of coherent models used in the DFT calculations above, in order to investigate the atomistic structure and thermodynamic stability at a larger length scale for commensurate interface models between unstrained $\mathrm{Ni}$ and $\alpha$-Fe.

There are no straightforward means to directly obtain the ground-state structure of a heterophase interface from an atomistic simulation. This is due to the difficulty to sufficiently sample a huge configuration space in a reasonable simulation time. Instead, one normally considers a subset of few representative interface models which are expected to be near to and exhibit similar characteristics as the true ground-state structure. One route to obtain such models is to choose some initial 
reference configuration based on an ideal lattice structure with a specific energetically favorable orientation relationship. This reference structure is subsequently optimized.

As the number of atoms in a $\mathrm{Ni}$ or $\mathrm{Fe}$ layer at or near the interface is not necessarily equal to that in the bulk volume, the simulated optimization procedure requires an effective algorithm that allows diffusion of atoms away from or towards the interface region. This is not readily possible in MD simulations with fixed number of atoms in bulk interfaces, because the displaced atoms would become interstitial, instead of reaching the surface for example. Also, diffusion processes occur on the time scale of seconds or minutes, and are difficult to access on the short time scale of atomic vibrations in molecular dynamics simulations.

Gumbsch et al. [1] used the vacancy formation energy in the region of the interface as a criterion for choosing the atoms which should be removed. Atoms with negative vacancy formation energies lead to a reduction of the total energy of the system when removed from the system and hence do not belong to the interface region. The authors demonstrated that a ground-state interface for a $\mathrm{Ni}-\mathrm{Ag}$ interface can be obtained by removing two [110] rows of atoms from the Ni side, which renders all vacancy formation energies positive, and gives rise to misfit dislocations at the interface.

Von Alfthan et al. [40] used a different approach, in which atoms were picked randomly from twist grain boundaries in Silicon, and the samples were subsequently annealed at various temperatures. In this way, the authors were able to find an ordered low-energy structure for the $\Sigma 5$ twist grain boundary, which was subsequently confirmed by first-principles DFT calculations.

Recently, Hashibon et al. [41] implemented a method which combines both the calculation of the vacancy formation energy profile and the "random" removal of atoms accompanied with annealing protocols to study the interface structure in $\mathrm{Cu}$-Ta systems. It was shown that both approaches lead to the same result for the ground-state interface structure, which was found to be characterized by a single $\mathrm{Cu}$ layer at the interface having mixed fcc-bcc features that facilitate the structural transition from bcc Ta to fcc $\mathrm{Cu}$ structures.

\subsubsection{Kinetic approach to ground-state interface models}

In the present work, we take a different, more straightforward approach which requires neither the random removal of atoms nor the calculation of the vacancy formation energy profiles to find ground state structures of ultra thin-film systems.

First, we choose two initial reference structures for $\mathrm{Ni}$ films on an $\alpha$-Fe (100) substrate. In the first one a bcc $\mathrm{Ni}$ film and in the second one a fcc Ni film are deposited on bcc Fe. The former represents one extreme case where the Ni film is in a state of complete coherency and compliance with the underlying structure of the Fe substrate. The latter represents the opposite extreme case, where Ni is in a state of complete incoherency and non-compliance. Naturally none of these reference structures corresponds a priori to a ground state, but both should be sufficiently close to it. The bcc structure is expected to be the ground state for a sufficiently thin film, or at least in the immediate vicinity of the bcc-Fe surface. The fcc structure is expected to be the ground state for a sufficiently thick film, at least sufficiently far away from the interface, where the structure in the film approaches that of the stable phase of bulk Ni.

Second, systems with varying numbers of Ni layers are introduced for each reference structure as starting configurations. The number of layers considered ranged between 1 and $25 \mathrm{fcc}$ or bcc layers of $\mathrm{Ni}$ on the bcc $\alpha$-Fe (001) substrate. This enables the analysis of the stability and structure as a function of the thickness of the film. 
Finally, each of the starting configurations is subject to annealing protocols at four different target temperatures: $T=300 \mathrm{~K}, 600 \mathrm{~K}, 900 \mathrm{~K}$, and $1200 \mathrm{~K}$. Although these temperatures are much lower than the melting points for bulk Ni, $T_{m}=1805 \mathrm{~K}$, and $\mathrm{Fe}, T_{m}=2135 \mathrm{~K}$, as calculated with the current interatomic potential [8], substantial thermal disorder is observed in the films during the simulations. In particular for $T>600 \mathrm{~K}$, strong disorder occured in few cases, destroying memory of the initial structures to a large extent. As expected [8], at higher temperatures a substantial mixing between $\mathrm{Fe}$ and $\mathrm{Ni}$ occurred, which we wanted to avoid as much as possible during the annealing. Hence the maximal temperature of the annealing protocol was not increased beyond $1200 \mathrm{~K}$. The equations of motion were integrated using the Nose-Hoover thermostat with a time step of $2 \mathrm{fs}$, for a total of $4.7 \mathrm{~ns}$, using the IMD program package [42]. At the end of a heating phase, the system is quenched, and the temperature is gradually reduced to room temperature. Finally, an energy minimization using conjugate gradients is performed at 0K. Each relaxation is carried out until the total forces on all atoms are less than $10^{-4} \mathrm{eV} / \mathrm{A}^{\circ}$. A total set of 64 different initial systems and temperature protocols was treated in this manner.

These simulations provide by no means a comprehensive sampling of the configuration space. But they do provide means for effectively inspecting the approach of the systems to its ground state, starting from different initial conditions. By careful comparison of the paths and the final relaxed systems, we obtain valuable information regarding the phase stabilities and the ground-state structures. If sufficiently annealed, it should be expected that all starting configurations will result in equivalent, or at least similar final structures having low energies.

\subsubsection{Initial interface models}

In addition to the coherent bcc/bcc interfaces from section 3.1, we consider now two orientation relationships for fcc $\mathrm{Ni}$ on $\alpha$-Fe (001) interfaces as initial configurations. The first orientation relationship (OR I) is a simple cube-on-cube system: $\mathrm{Ni}(001)\langle 100\rangle / / \alpha-\mathrm{Fe}(001)\langle 100\rangle$, schematically shown in Figure 2(a). The second orientation relationship (OR II) is the one reported by experimental studies [1214, 14]: $\mathrm{Ni}(110)\langle 1 \overline{1} 2\rangle / / \alpha-\mathrm{Fe}(001)\langle 110\rangle$, as schematically shown in Figure 2(b). In addition to the surface unit cells, few of the nearest neighbor (NN) distances along high symmetry directions are indicated on the figure.

The $\alpha$-Fe substrates are composed of up to 60 bcc (001) layers, the positions of the lowest 4 layers of the Fe were fixed. The top 1 to 25 layers were substituted with layers of $\mathrm{Ni}$ in either coherent bcc configurations at the same orientation or incoherent fcc configurations corresponding to either OR I or OR II. System sizes of up to approximately 50,000 atoms were used.

Commensurate interfaces for the fcc-Ni/bcc-Fe incoherent systems are constructed such that negligible misfit strains exist in the initially fcc $\mathrm{Ni}$ films and bcc Fe substrates. For OR I, $22 \times 22 \mathrm{fcc}(001) \mathrm{Ni}$ surface unit cells are fit to $27 \times 27$ bcc (001) Fe surface unit cells, resulting in misfit strain of less than $0.05 \%$. For OR II, $16 \times d_{[211]}$ and $6 \times d_{[111]}$ Ni planes were matched to $17 \times d_{[110]}$ and $9 \times d_{[111]} \mathrm{Fe}$ planes ( $d$ is the interplanar spacing). In the case of a bcc-film system, the strain in the film will eventually lead to an instability of the system and cause a structural transformation, resulting in the formation of either a bcc, bct, or another intermediate distorted fcc phase. In the incoherent case the restructuring of the film is expected to be driven by the incoherency, which results in certain atoms to be at unfavorable on-top positions. In both cases, the initial number of atoms per layer near the interfaces is in general not equal to that for the ground state. This will lead to an enhanced restructuring and diffusion, which is expected to reveal certain trends in the approach of the system towards equilibrium. 


\subsubsection{Structural transformations in thin films}

The result for the annealing of the fcc system with $1 \mathrm{ML}$ of $\mathrm{Ni}$ on Fe is demonstrated in Figure 3 where [010] projections of the initial, unrelaxed system, the relaxed configuration at $0 \mathrm{~K}$, and the relaxed configurations resulting after annealing at the various temperatures, are shown. The fcc Ni ML illustrates restructuring already at $0 K$ as it can be seen by comparing the initial unrelaxed system in Figure 3(a) and the system after relaxation in Figure 3(b). This restructuring leads to the formation of small islands on top of a transformed first layer. As the excess atoms are expelled from the fcc layer, the remaining atoms transform the film into a bcc structure. Hence, the initial fcc ML structure of the Ni film results in a transformation to a bcc ML structure with additional islands adsorbed on top. In the corresponding system of a bcc ML of $\mathrm{Ni}$ on a bcc Fe, the layer remained bcc until the highest annealing temperature was reached, where mixing was observed. That is, the pseudomorphic bcc Ni ML is stable up to $900 K$. Only at the highest considered temperature of $1200 K$ does the Ni layer get completely absorbed into the Fe substrate.

For the systems with initially 1-3 ML of bcc Ni, no structural transitions occurred, apart from a change in the interplanar separation normal to the interface. The models with initially 1-3 ML of fcc Ni, on the other hand, do transform. Figure 4, shows a [010] projection of the initial and final relaxed and annealed systems for $3 \mathrm{ML}$ of fcc $\mathrm{Ni}$ on bcc Fe. The fcc-Ni film remains stable against relaxation at $0 \mathrm{~K}$ and annealing at $300 \mathrm{~K}$, but transforms after annealing at $600 \mathrm{~K}$ and above into a bcc-Ni phase.

In both the initial bcc films and the transformed fcc films for the systems with 1-3 ML of $\mathrm{Ni}$, the interplanar separation in the ground-state $\mathrm{Ni}$ films is found to be $d=1.38 \mathrm{~A}$, in accordance with the value found experimentally $d=1.35 \AA$ by Kamada and Matsui [12]. Figure 5 shows a [001] projection (a Moiré pattern) of the first $\mathrm{Ni}$ layer (gray) on an ideal Fe bcc (001) layer (black). The Ni atoms are located on almost the same positions as the Fe atoms underneath, i.e., the film has a bcc (001) structure. The average in-plane nearest-neighbor (NN) distance is found to be $a=2.7 \mathrm{~A}$, smaller than $a=2.81 \mathrm{~A}$ of a bcc-Ni phase, with a ratio $c / a=1.022$. Hence the structure is body-centered tetragonal (bct) which is only slightly distorted from the cubic bcc structure.

The systems with initially 4 bcc-Ni ML also show no transformation of the bccNi structure, but do show a network of misfit dislocations near the interface region. The system with initially 4 fcc-Ni ML transforms to the bcc structure, also with a network of misfit dislocations. These dislocations at the interfaces will be further discussed elsewhere.

\subsubsection{Excess interface energy}

For each of the systems, the excess interface energy $\gamma$ is calculated according to equation 3. The results are shown in Table 4 for the first $4 \mathrm{ML}$ for all systems after annealing, and in Figure 6 for bcc $\mathrm{Ni}$ and OR I as a function of the initial number of layers in the systems. The dashed lines in the figure connect data points for the systems with initial fcc-Ni structures, and solid lines for the initial bcc-Ni ones. The chemical potential of Fe in equation 2 is taken from the bulk region in the center of the substrate, while for $\mathrm{Ni}$, the cohesive energy of relaxed bulk crystal is used. The excess energy $\gamma$ includes the excess energy due to the Fe-Ni interface, the deviation of Ni from the fcc structure (i.e., strain energy), the free surface energy of the $\mathrm{Ni}$ film, and any island formation or additional defects in the system. Only the energies of the relaxed and annealed systems are shown. Also indicated in the figure is the surface energy of the free bcc (001) Fe surface $\left(\gamma=2.17 \mathrm{~J} / \mathrm{m}^{2}\right)$ by an 
arrow on the abscissa.

There are clearly two distinct regimes of the behaviour of the excess interface energy of OR I in Figure 6. In the first regime, below 3-4 ML, both the fcc and the bcc initial systems have relatively low interface excess energies, which are almost equal, except for the systems with 3 and 4 initial fcc-Ni ML at 0K and 300K (the ' $\mathrm{x}$ ' and ' + ' symbols in Figure 6), for which the annealing did not result in a complete transformation. In fact, if the fcc curve (dashed lines) is shifted by one ML unit to the right along the x-axis, the dashed and full curves almost coincide in this regime. This is a result of the structural transformation in these systems as demonstrated in Figure 3 for the fcc $1 \mathrm{ML}$ and Figure 4 for the fcc $3 \mathrm{ML}$ films, where an additional layer was generated during the transformation from fcc to bcc (or bct with $\mathrm{c} / \mathrm{a} \approx 1$ ). This is because the fcc-Ni layers have excess of atoms with respect to the bcc-Fe substrate (968 in the fcc (001) Ni compared to 729 in the bcc (001) Fe). The compliance of thin films of up to $4 \mathrm{ML}$, drives the excess atoms of the initially perfect fcc layers to move out of the interface region and form new adlayers or islands. The remaining atoms at the interface get restructured into a bcc phase.

The instability of the $1 \mathrm{ML}$ fcc-Ni on the bcc-Fe (001) substrate shown in Figure 3 is of course expected, and it can also be seen from the excess interface energy $\gamma$ for $1 \mathrm{ML}$ shown in Figure 6 (the first point on the dashed line, with a '+' symbol), where the excess energy of the relaxed fcc ML at $0 \mathrm{~K}, \gamma=2.22 \mathrm{~J} / \mathrm{m}^{2}$, is larger than that of $1 \mathrm{ML}$ of bcc Ni, $\gamma=1.844 \mathrm{~J} / \mathrm{m}^{2}$. By inspecting the energies of the various systems for $1 \mathrm{ML}$ in Figure 6, it is evident that apart from the fcc ML at $0 \mathrm{~K}$ the excess energies of a single Ni layer, at all temperatures for both fcc and bcc films, are significantly lower than the free surface energy of $\alpha$-Fe (001) (indicated by an arrow at the y-axis in Figure 6). Hence, a ML of $\mathrm{Ni}$ on $\mathrm{Fe}$ is thermodynamically stable and completely covers the Fe surface.

Adding a second bcc or fcc ML of Ni leads to a relatively small increase of the excess interface energy, of the order of $0.1-0.3 \mathrm{~J} / \mathrm{m}^{2}$, as seen from Figure 6 and Table 4. This increase is not large enough to render two or more ML unstable, since it is of the same size as for adlayers (cf. the energies of the annealed OR I systems which do contain adlayers, with the bcc systems which do not in Figure 6.).

Notice that our method, since it does not account for the removal of atoms, may not be sensitive enough to reveal whether a layer-by-layer growth mode is favorable, although a similar approach with a similar potential [39] did lead to dewetting for the $\mathrm{Cu}$-Ta system. Nevertheless, our goal in the present work is to investigate structural trends in the layers adjacent to the interface. Hence the excess energies, which include additional defects, are not most appropriate to determine the thermodynamic stability of the films.

As seen in Figure 6, for more than 3-4 ML the excess energy of the bcc systems increases linearly with the number of ML for all temperatures. Assuming this increase is a result of strain, the slope of the line is, by definition, the excess strain energy per layer in the system. In the same region, the fcc phase seems stable with respect to the annealing protocols used here, since the excess energy becomes nearly constant. This implies that no additional contributions to $\gamma$ exist apart from those arising from the interface energy and from the free surface energy of $\mathrm{Ni}$, which does not depend on the number of ML in between (their energy is bulk like, and hence cancel out, cf. Equation 3). We cannot exclude the possibility that these systems are already too large and in such deep local minima, that the annealing protocols used here were too short in time to allow for any transformation. In fact, for the fcc systems with 5 and more ML, it was apparent that the high energies of the $\mathrm{Ni}$ atoms at the interface induced an enhanced mixing of $\mathrm{Ni}$ and $\mathrm{Fe}$ at the interface, 


\section{Page 11 of 23

and the formation of a fcc Fe-Ni phase on the Fe side. Adding more Ni layers to this system did not change the interface region, therefore we neglect these systems. Nevertheless, it is rather surprising that the switch in behaviour occurred both for the fcc and bcc initial configurations at the same thickness of 3-4 ML which is also observed in experiments [11-15, 17].

\subsubsection{In-plane structural relationships}

We next take a closer look at the in-plane structure of the $3 \mathrm{ML}$ fcc-Ni system. The in-plane structure is investigated via the two-dimensional radial distribution function (2DRDF) calculated for each layer, which is the same as the usual RDF, (cf., e.g., Ref. [43]), except that each layer is considered as a two-dimensional structure with projected distances on the plane of the layer. The 2DRDF gives information about the neighbour shells within each layer. Hence it can directly reveal structural relations and trends before the actual structure has even emerged, i.e., before directional order sets in.

The 2DRDF of the Ni layer directly on top of the substrate in the ground-state of the system with initially $3 \mathrm{fcc}-\mathrm{Ni} \mathrm{ML}$ is shown in Figure 7, together with the 2DRDF of ideal bcc-Fe (001), fcc-Ni (001), and fcc-Ni (110) planes, marked on the figure by solid, dashed, and dotted lines, respectively. The NN shells marked by "A" and "B" on Figure 7 exist only in the fcc-Ni (110) plane, while the NN shell marked "C" on the figure exist only in the fcc-Ni (001) plane. We note first that in spite of the compliance of $\mathrm{Ni}$ to the bcc structure of Fe, as evident from the (001) projection in Figure 5, the layer has a distorted bcc in-plane structure with many interatomic distances close to those in fcc Ni. These distortions are seen from the shifts of the NN distances in the 2DRDF in Figure 7. The position of the first peak in the Ni layer in Figure 7 shifts towards a larger value, closer to that of the first NN distance in a bulk bcc (001) Fe plane, indicating a line matching along $\langle 100\rangle$ directions. The peak corresponding to the third NN distance of fcc-Ni (001) planes $\left(d_{3 N N}=4.98 \AA\right)$, is completely absent from the 2DRDF of the Ni layer. Instead there is a peak near the bcc $(001)$ second $\mathrm{NN}$ distance $\left(d_{2 N N}=4.05 \AA\right)$. However this peak is also close to the third NN distance in fcc-Ni (110) planes (at $4.3 \AA$, cf. Figure 2(b)). This indicates line matching along the $\langle 112\rangle$ in fcc Ni directions, corresponding to the $\langle 110\rangle$ directions in bcc Fe. Finally, a peak in the 2DRDF of Ni is also found at the fifth NN distance (at $6.1 \AA$, cf. Figure $2(\mathrm{~b})$ ), which corresponds to the $\langle 111\rangle$ direction in a fcc (110) Ni layer.

These interface-specific peaks marked A and B in Figure 7 and the shifts in the second NN distances, indicate a possible preferred direction for line matching between fcc $\mathrm{Ni}$ and bcc Fe, which is independently obtained from the bcc/bcc interfaces and from those of fcc Ni on bcc Fe in OR I. In particular, the two peaks $\mathrm{A}$ and $\mathrm{B}$ are typical of a (110) fcc-Ni plane, with the $\langle 1 \overline{1} 2\rangle$ fcc-Ni direction parallel to the $\langle 1 \overline{1} 0\rangle$ bcc-Fe direction, which is exactly the orientation OR II. Hence by starting with either OR I, or coherent bcc/bcc interfaces, the trends in the 2DRDF suggest that OR II is more favourable.

We note that the 2DRDF gives only averaged distances of neighbouring shells and does not indicate directional order. Hence, although the nearest-neighbour distances may be compatible with those of a (110) plane, the interface may not necessarily be of (110) type, as seen by comparing Figures 5 and 7 . In fact, such a transformation will not appear since the size of the system is constrained to that of OR I. Indeed for the thicker films, large strains are found in the systems, as also indicated by the regime of linear increase in Figure 6 for the bcc systems.

The OR II systems are already constructed such that $\langle 1 \overline{1} 2\rangle$-fcc is parallel to $\langle 110\rangle$-bcc. Figures 8 and 9 demonstrate similar but more pronounced features in 
OR II as compared to OR I. For the case of the 2 ML system, shown in Figure 8, the $\mathrm{Ni}$ film is transformed into bcc, where the first as well as the second NN distances are very close to those of bcc Fe. For the 4 ML case shown in Figure 9, the second NN distance is now larger than that in bcc Fe, and it is close to that found in fcc (110) Ni planes. Our results indicate therefore that for thicker Ni layers, a transition from bcc-Ni to fcc-Ni with OR II should occur. This is in agreement with findings in Refs. [14, 15], where X-ray diffraction experiments on OR I samples confirmed the transition to OR II. Hence, we have shown here that the kinetic approach to ground-state structures and the $2 \mathrm{DRDF}$ provide a simple but powerful route to determining orientation relationships in heterophase interfaces.

\section{Conclusion}

We have performed atomistic simulations for coherent $\mathrm{Fe} / \mathrm{Ni}$ interfaces and for thin Ni films on the bcc $\alpha$-Fe (001) substrates, using both DFT-LDA and atomistic simulations with an angle-dependent embedded-atom-method potential. Our results indicate that the used EAM (or Finnis-Sinclair type) potentials [8] with additional angle-dependent terms describes the interface adhesion of Ni-Fe systems for coherent interfaces in good agreement with ab-initio calculations. We then attempted to study the structural trends in thin films by means of kinetic simulations, without the need to calculate vacancy formation profiles or random removal of atoms. We demonstrated that this procedure, although it is not rigorous and has its limitations, can nevertheless be quite effective in identifying the favourable orientation relationship of incoherent interfaces by inspection of the main trends along the relaxation paths of different initial structures. The favourable orientation relationship which we obtained is also observed experimentally. We also found that up to 3-4 layers thin Ni films on Fe are stable in a slightly distorted bcc structure, in agreement with experiments, and that they undergo a transition to the fcc structure with increasing number of layers. We demonstrated that the transition occurs gradually by changing the first and third nearest neighbour distances in fcc Ni.

\section{Acknowledgments}

We gratefully acknowledge the financial support from the German Research Foundation DFG, Grant no. GU367/24-1, and from the German Federal Ministry for Education and Research BMBF, Grants no. 03X0511 and 01R105273. We would like to thank Yuri Mishin for providing to us the interatomic potential [8] used in this work.

\section{References}

[1] P. Gumbsch, M.S. Daw, S.M. Foiles and H.F. Fischmeister, Phys. Rev. B 43 (1991) p.13833.

[2] P. Gumbsch, Z. Metallkd. 83 (1992) p.500.

[3] L.B. Freund and S. Suresh Thin Film Materials: Stress, Defect Formation and Surface Evolution, Cambridge University Press, 2003.

[4] J. Howe Interfaces in Materials, Wiley-Interscience Publication, 1997.

[5] M. Wuttig and X. Liu Ultrathin Metal Films, Springer-Verlag, Berlin, 2004.

[6] C.A.F. Vaz, J.A.C. Bland and G. Lauhoff, Rep. Prog. Phys. 71 (2008) p.056501.

[7] V.L. Moruzzi, P.M. Marcus, K. Schwarz and P. Mohn, Phys. Rev. B 34 (1986) p.1784.

[8] Y. Mishin, M. Mehl and D. Papaconstantopoulos, Acta Mater. 53 (2005) p.4029.

[9] B. Heinrich, A. S. Arrott, J. F. Cochran, S. T. Purcell, K. B. Urquhart and K. Myrtle, J. Cryst. Growth 81 (1987) p.562.

[10] B. Heinrich, S.T. Purcell, J.R. Dutcher, K.B. Urquhart, J.F. Cochran and A.S. Arrott, Phys. Rev. B 38 (1988) p.12879.

[11] Z. Wang, S. Lu, Y. Li, F. Jona and P. Marcus, Phys. Rev. B 35 (1987) p.9322.

[12] Y. Kamada and M. Matsui, J. Phys. Soc. Jpn. 66 (1997) p.658.

[13] Y. Kamada, M. Matsui and T. Asada, J. Phys. Soc. Jpn. 66 (1997) p.466. 


\section{Page 13 of 23 \\ Philosophical Magazine \& Philosophical Magazine Letters}

[14] A.V. Mijiritskii, P.J.M. Smulders, V.Y. Chumanov, O.C. Rogojanu, M.A. James and D.O. Boerma, Phys. Rev. B 58 (1998) p.8960.

[15] A. Mijiritskii and D. Boerma, J. Magn. Magn. Mater. 232 (2001) p.9.

[16] C.J. Gutierrez, M.D. Wieczorek, Z.Q. Qiu, H. Tang and J.C. Walker, J. Magn. Magn. Mater. 93 (1991) p.369.

[17] T. Lin, M.M. Schwickert, M.A. Tomaz, H. Chen and G.R. Harp, Phys. Rev. B 59 (1999) p.13911.

[18] P. Hohenberg and W. Kohn, Phys. Rev. 136 (1964) p.864.

[19] W. Kohn and L.J. Sham, Phys. Rev. 140 (1965) p.A1133.

[20] D.M. Ceperley and B.J. Alder, Phys. Rev. Lett. 45 (1980) p.566.

[21] J.P. Perdew and A. Zunger, Phys. Rev. B 23 (1981) p.5048.

[22] D.R. Hamann, M. Schlüter and C. Chiang, Phys. Rev. Lett. 43 (1979) p.1494.

[23] S.G. Louie, K.M. Ho and M.L. Cohen, Phys. Rev. B 19 (1979) p.1774.

[24] C.L. Fu and K.M. Ho, Phys. Rev. B 28 (1983) p.5480.

[25] C. Elsässer, N. Takeuchi, K.M. Ho, C.T. Chan, P. Braun and M. Fähnle, J. Phys.: Condens. Matter 2 (1990) p. 4371.

[26] B. Meyer, K. Hummler, C. Elsässer and M. Fähnle, J. Phys. Condens. Matter, 7 (1995) p.9201.

[27] B. Meyer, C. Elsässer, F. Lechermann and M. Fähnle, Fortran90 Program for Mixed-Basis Pseudopotential Calculations for Crystals, Max-Planck-Institut für Metallforschung Stuttgart.

[28] F. Lechermann, F. Welsch, C. Elsässer, C. Ederer, M. Fähnle, J.M. Sanchez and B. Meyer, Phys. Rev. B 65 (2002) p.132104.

[29] F. Lechermann, M. Fähnle, B. Meyer and C. Elsässer, Phys. Rev. B 69 (2004) p.165116.

[30] D. Vanderbilt, Phys. Rev. B 32 (1985) p.8412.

[31] H.J. Monkhorst and J.D. Pack, Phys. Rev. B 13 (1978) p.5747.

[32] A. Hashibon, J. Adler, M. Finnis and W. Kaplan, Interface Sci. 9 (2001) p.175.

[33] A. Hashibon, J. Adler, M. Finnis and W. Kaplan, Comput. Mater. Sci. 24 (2002) p.443.

$34]$ A. Hashibon, C. Elsässer and M. Rühle, Acta Mater. 53 (2005) p.5323.

[35] A. Hashibon, C. Elsässer, Y. Mishin and P. Gumbsch, Phys. Rev. B 76 (2007) p.245434.

[36] M. Finnis, J. Phys.: Condens. Matter 8 (1996) p.5811.

[37] A. Hashibon, C. Elsässer and M. Rühle, Acta Mater. 55 (2007) p.1657.

[38] J.H. Rose, J.R. Smith, F. Guinea and J. Ferrante, Phys. Rev. B 29 (1984) p.2963.

[39] A. Hashibon, A.Y. Lozovoi, Y. Mishin, C. Elsässer and P. Gumbsch, Phys. Rev. B 77 (2008), p.094131.

[40] S. von Alfthan, P.D. Haynes, K. Kaski and A. P. Sutton, Phy. Rev. Lett. 96 (2006) p.055505.

[41] A. Hashibon, C. Elsässer and P. Gumbsch, in Proc. 17th Plansee Seminar, Reutte, Austria vol. 3 (2009) p.WS41.

[42] J. Stadler, R. Mikulla and H. Trebin, Int. J. Mod. Phys. C 8 (1997) p.1131.

[43] D.C. Rapaport, The Art of Molecular Dynamics Simulation - 2nd ed., Cambridge University Press, 2004.

[44] C. Kittel, Introduction to Solid State Physics,, Wiley-Interscience, New York, 1986.

[45] J. Zarestky and C. Stassis, Phys. Rev. B 35 (1987) p.4500. 


\begin{tabular}{|cl|ccc|}
\hline Substrate & $\mathrm{Film}$ & $\epsilon_{x}$ & $\epsilon_{y}$ & $\epsilon_{z}$ \\
\hline$\alpha-\mathrm{Fe}(110)$ & $\mathrm{Ni}(111)$ & 11.5 & -8.9 & -0.89 \\
$\alpha-\mathrm{Fe}(110)$ & $\mathrm{Cr}(110)$ & -3.1 & -3.1 & 3.46 \\
$\gamma-\mathrm{Fe}(111)$ & $\mathrm{Ni}(111)$ & -1.2 & -1.2 & 0.48 \\
$\gamma-\mathrm{Fe}(111)$ & $\mathrm{Cr}(110)$ & -14.1 & 5.18 & 6.66 \\
\hline
\end{tabular}

Table 2. Residual strains in the coherent interface models obtained by comparing the energies of the coherent models and ideal bulk crystals obtained from the DFT-LDA calculations.

\begin{tabular}{|cccc|}
\hline Interface model & $W_{\text {sep }}$ & $\gamma_{\epsilon}$ & $\Delta$ \\
& $J / m^{2}$ & $J / m^{2}$ & $\AA$ \\
\hline$\alpha-\mathrm{Fe}(110)-\mathrm{Ni}(111)$ & 5.5707 & 0.25 & 3.69 \\
$\alpha-\mathrm{Fe}(110)-\mathrm{Cr}(110)$ & 6.8500 & 0.06 & 3.72 \\
$\gamma-\mathrm{Fe}(111)-\mathrm{Ni}(111)$ & 5.6885 & 0.0024 & 3.78 \\
$\gamma-\mathrm{Fe}(111)-\mathrm{Cr}(110)$ & 6.5945 & 1.05 & 3.89 \\
\hline
\end{tabular}

Table 3. The ideal work of separation, excess strain energy per monolayer (ML), and equilibrium interface separation for the coherent interface models obtained from DFT-LDA calculations.

\begin{tabular}{cccc}
\hline ML & bcc & OR I & OR II \\
\hline 1 & 1.84 & 1.97 & 1.91 \\
2 & 2.13 & 2.25 & 2.18 \\
3 & 2.28 & 2.40 & 2.30 \\
4 & 2.36 & 2.56 & 2.42 \\
\hline
\end{tabular}

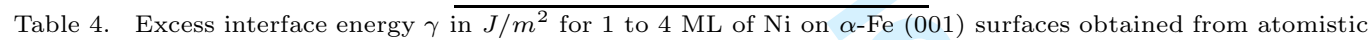
EAM-ADP simulations. Only the energies of ground-state interface structures are given. 
(a)

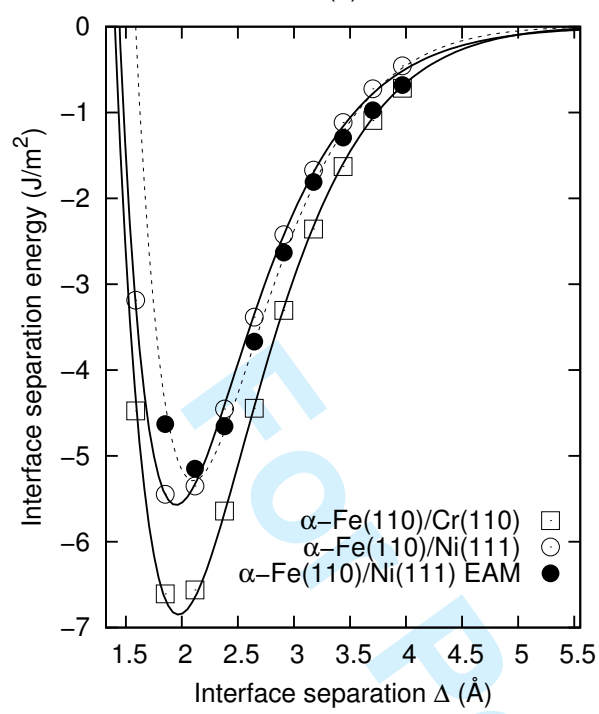

(b)

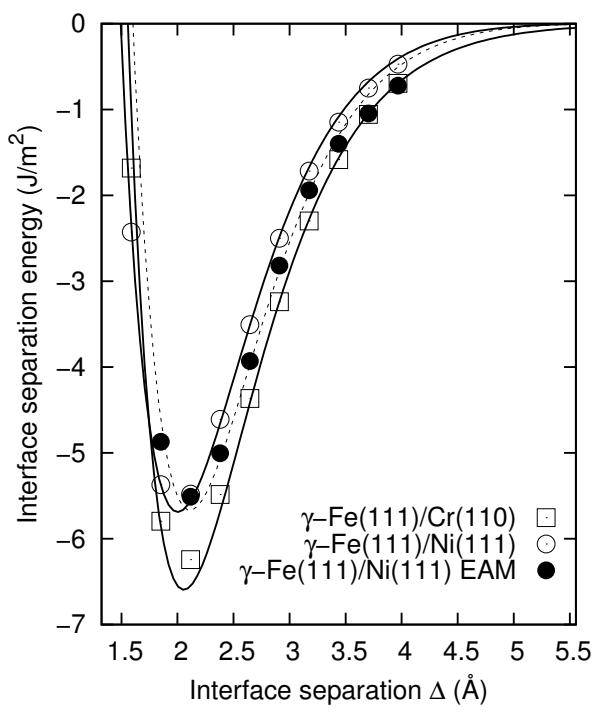

Figure 1. The interface separation energy curves for bcc Cr and fcc Ni on bcc $\alpha$-Fe (left panel) and fcc $\gamma$-Fe (right panel) from DFT-LDA calculations (open symbols) and EAM-ADP simulations (filled symbols). 
(a)

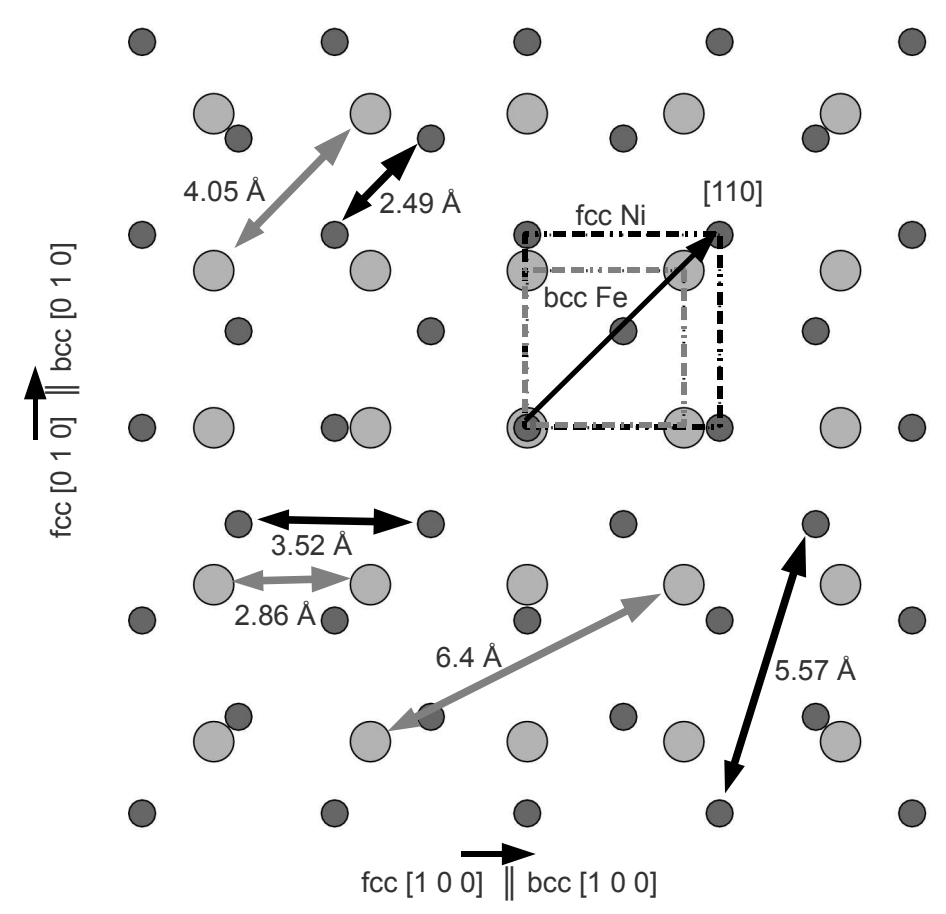

(b)

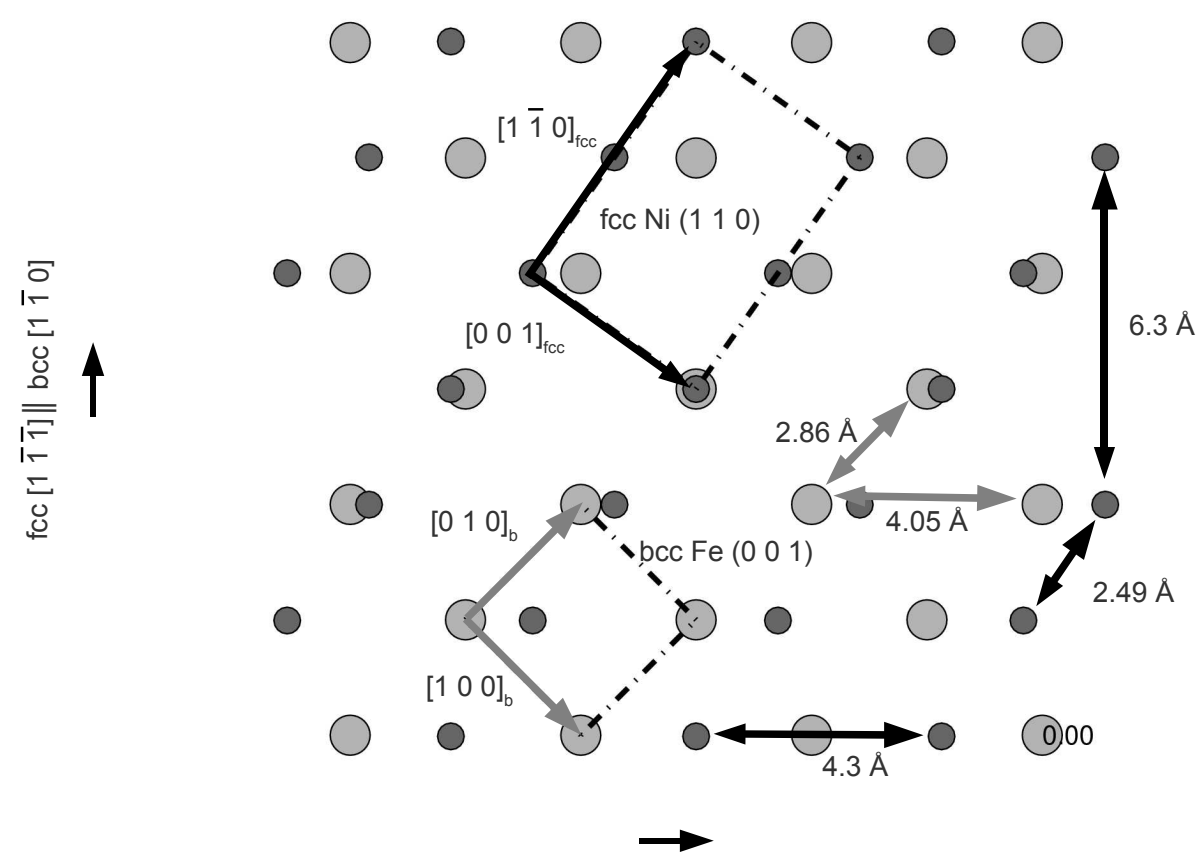

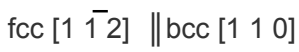

Figure 2. A schematic representation of the interface structure for interface orientation relationships (a) OR I, and(b) OR II. The surface unit cells of fcc-Ni and bcc-Fe are shown in the figure. The numbers state the distances in special symmetry directions. See text for more details. 
3 4

5

7

8

9

10

11

12

13

14

15

16

17

18

19

20

21

22

23

24

25

26

27

28

29

30

31

32

33

34

35

36

37

38

39

40

41

42

43

44

45

46

47

48

49

50

51

52

53

54

55

56

57

58

59

60

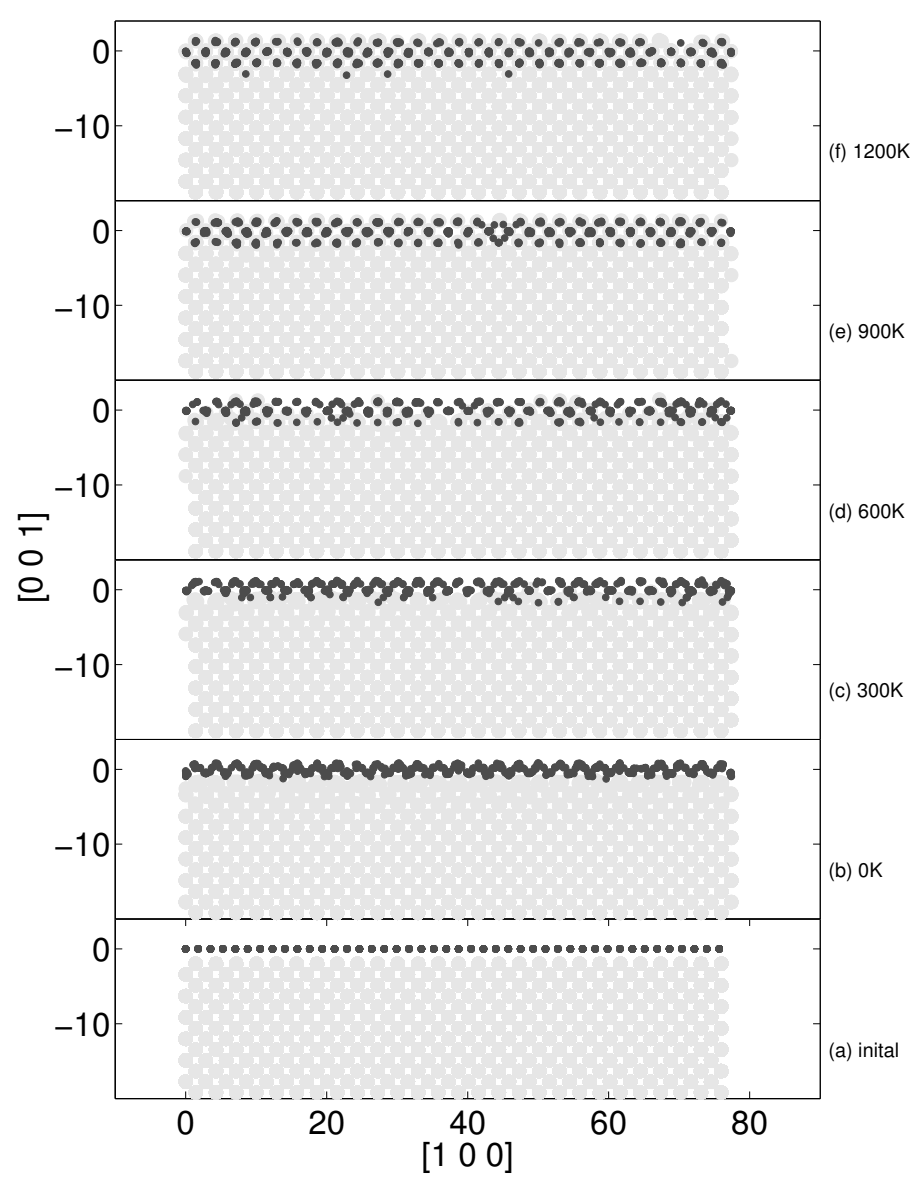

Figure 3. A view of systems with $1 \mathrm{ML}$ of fcc $\mathrm{Ni}$ on the (001) surface of bcc Fe in ORI projected along [010]: (a) the initial unrelaxed system, (b) the relaxed configuration at 0K, and (c-d) the relaxed configurations resulting after annealing at the various temperatures, as indicated on the Figure. As a result of the annealing, already at $\mathrm{T}=300 \mathrm{~K}$ (c) small islands form on the surface. At high temperatures (d-f), Ni atoms are driven into the Fe sublattice to lower energy, and strong mixing of $\mathrm{Ni}$ and Fe occurs. 




Figure 4. A view of systems with $3 \mathrm{ML}$ of fcc Ni on the (001) surface of bcc Fe in ORI projected along [010]: (a) the initial unrelaxed system, (b) the relaxed configuration at $0 \mathrm{~K}$, and (c-d) the relaxed configurations resulting after annealing at the various temperatures, as indicated on the Figure. The annealing enables the fcc-Ni layer to transforms into a bcc-Ni layer (d-f), reducing the planar density, and resulting in an additional incomplete bcc layer. 

4 5 7

8

9

10

11

12

13

14

15

16

17

18

19

20

21

22

23

24

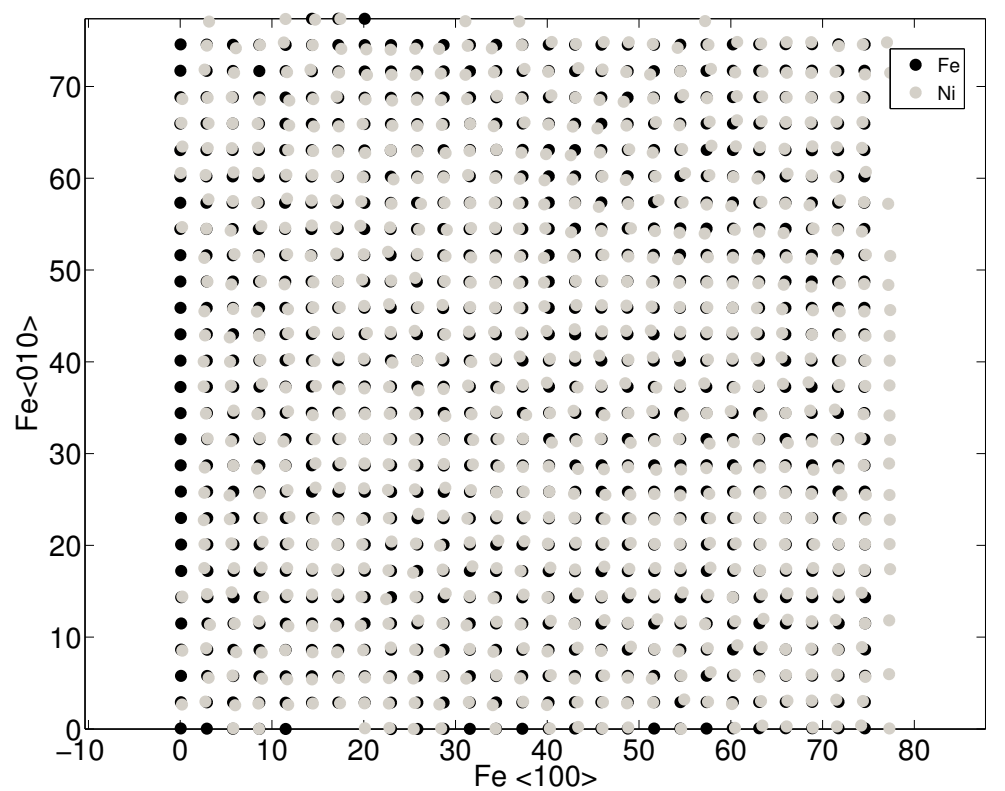

Figure 5. The structure of the Ni layer directly at the interface in the ground state of the system with initially $3 \mathrm{ML}$ of fcc $\mathrm{Ni}$, shown in a projection along the normal direction to the interface $\langle 001\rangle$. The $\mathrm{Ni}$ layer (grey symbols) is superimposed on the bcc-Fe (001) layer (Black symbols). The atoms of the Ni are found almost directly on top of the bcc-Fe (001) positions, indicating a bcc structure of the Ni layer in the ground state. 


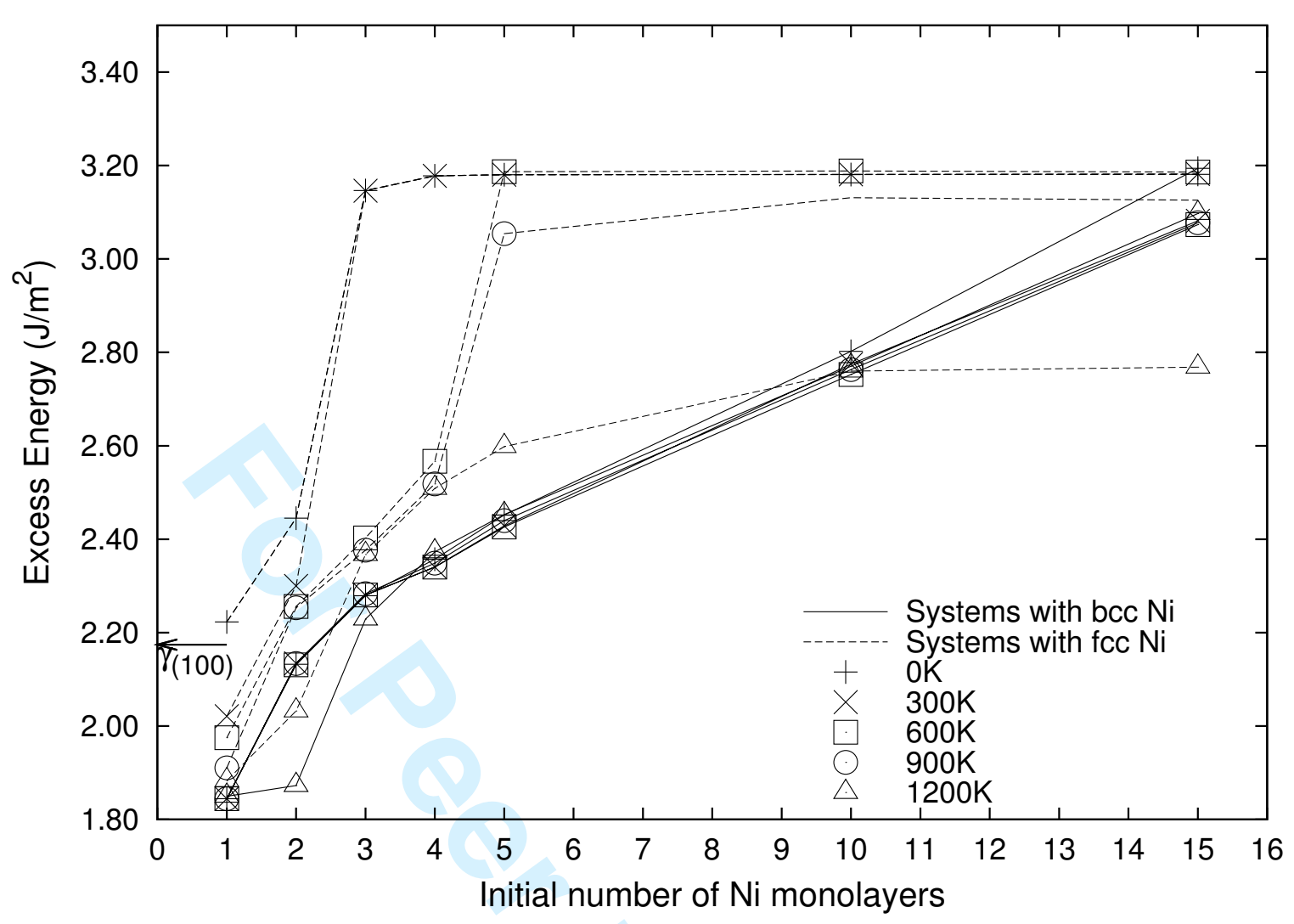

Figure 6. The excess interface energy $\gamma$ as a function of the initial number of fcc (dashed lines) and bcc (solid lines) $\mathrm{Ni}$ on $\mathrm{Fe}(001)$ in OR I. The arrow on the abscissa indicates the energy of a free $\alpha$-Fe(001) surface. Two regions are clearly visible, below and above 3-4 ML. Above the excess interface energy increases linearly for the bcc systems while it is nearly constant for the fcc systems. 
1

2

3

4

5

6

9

10

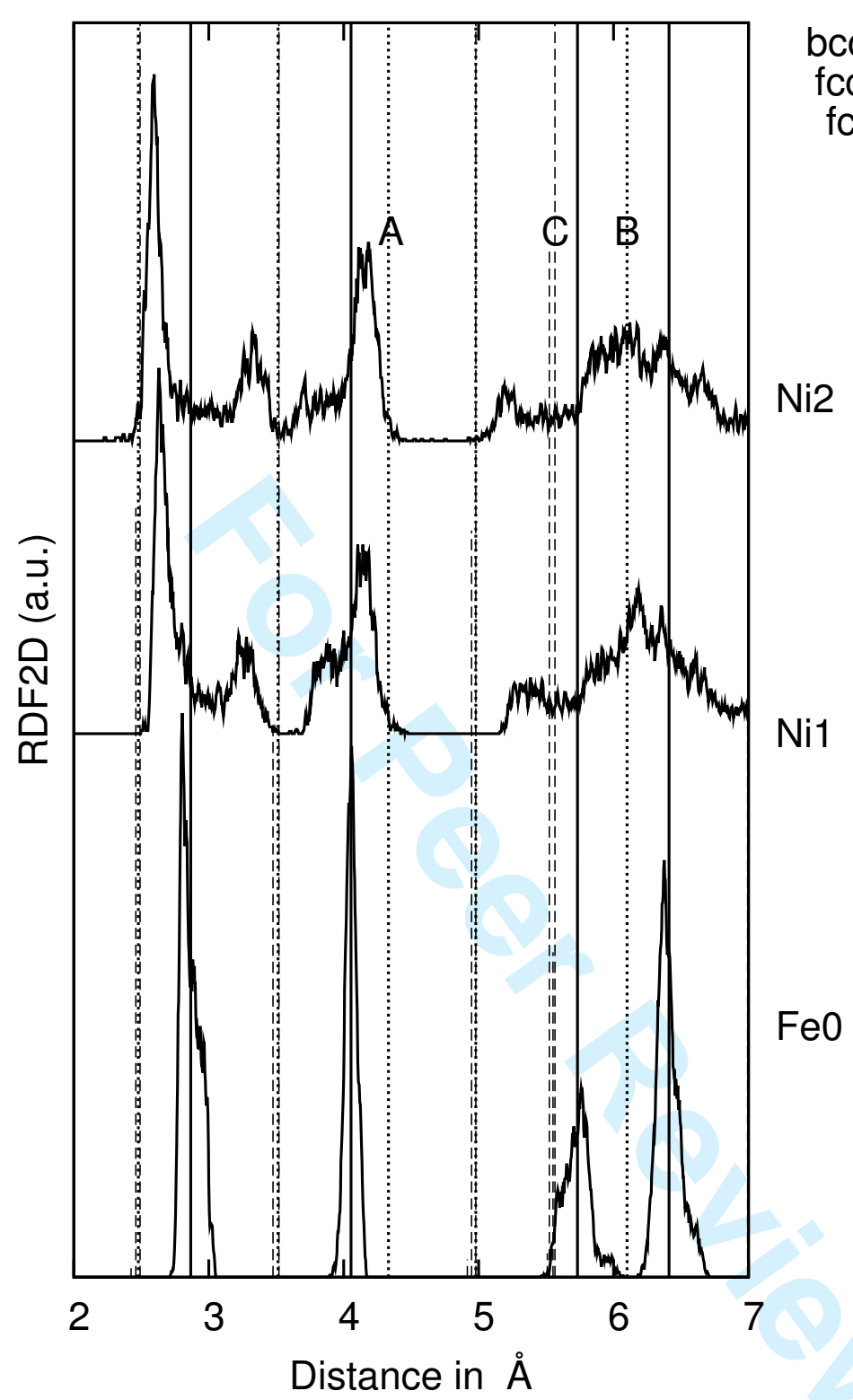

Figure 7. The 2DRDF for layers directly at the interface in the ground-state structure of the system with initially $3 \mathrm{ML}$ of fcc Ni in OR I, shown in Figure 5. The peaks of the fcc-Ni are common for both fcc-Ni (001) and fcc-Ni (110) planes, except of the peaks marked A and B which only belong to the fcc-Ni (110) plane, and the peak marked $\mathrm{C}$ which only belong to the fcc-Ni (001) plane. Fe0 is the top-most Fe layer of the substrate. Ni1 and Ni2 are the first two Ni layers at the interface. 


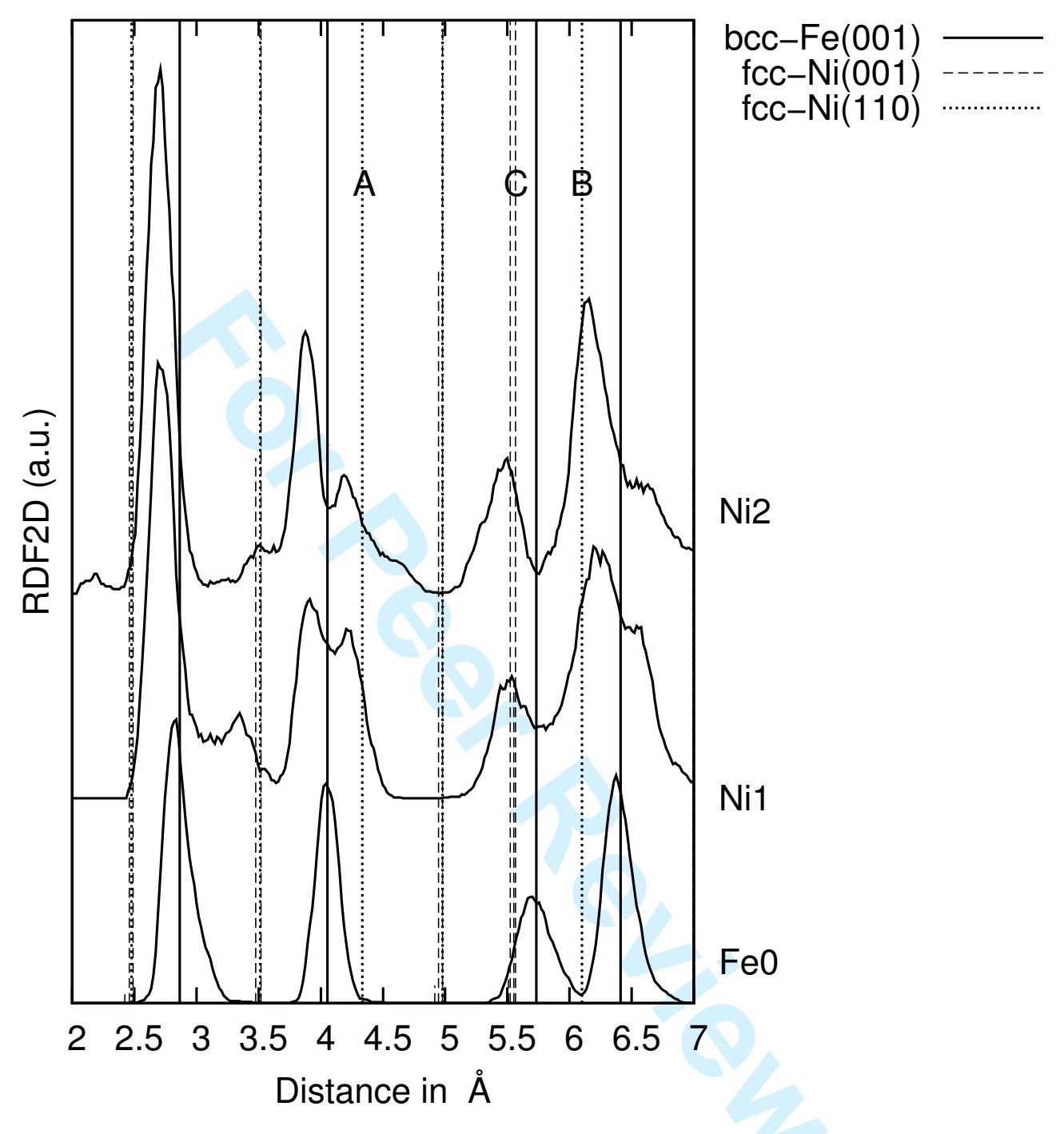

Figure 8 . The $2 \mathrm{DRDF}$ for the layers directly at the interface in the ground-state structure of the system with initially $2 \mathrm{ML}$ of fcc $\mathrm{Ni}$ in OR II. The peaks of the fcc-Ni are common for both fcc-Ni (001) and fcc-Ni (110) planes, except of the peaks marked A and B which only belong to the fcc-Ni (110) plane, and the peak marked $\mathrm{C}$ which only belong to the fcc-Ni (001) plane. $\mathrm{Fe} 0$ is the top-most Fe layer of the substrate. $\mathrm{Ni} 1$ and $\mathrm{Ni} 2$ are the first two Ni layers at the interface. 


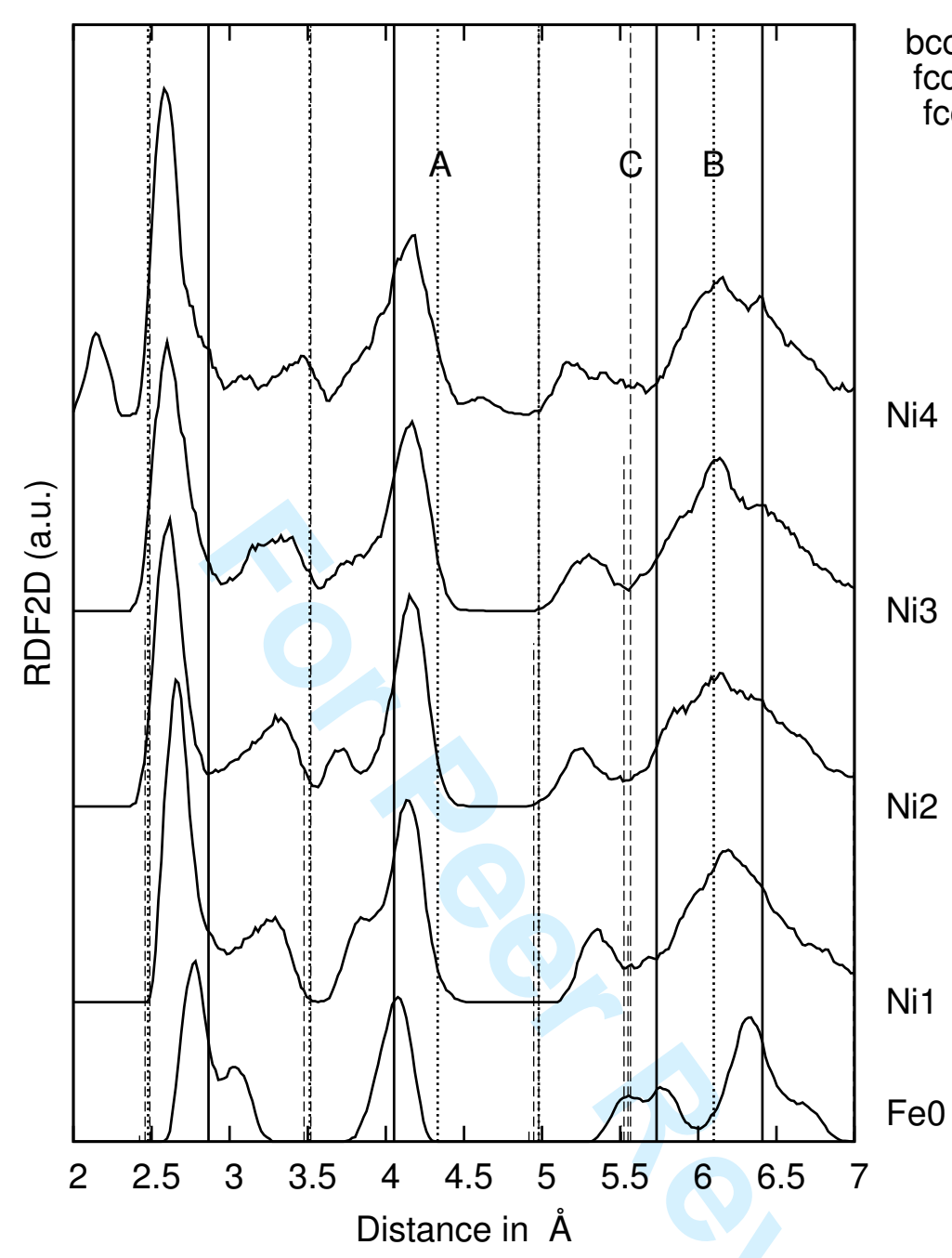

Figure 9. The 2DRDF for the layers directly at the interface in the ground-state structure of the system with initially $4 \mathrm{ML}$ of fcc $\mathrm{Ni}$ in OR II. The peaks of the fcc-Ni are common for both fcc-Ni (001) and fcc-Ni (110) planes, except of the peaks marked A and B which only belong to the fcc-Ni (110) plane, and the peak marked $\mathrm{C}$ which only belong to the fcc-Ni (001) plane. Fe0 is the top most layer of the substrate. Ni1 to Ni4 are the first four Ni layers at the interface. 